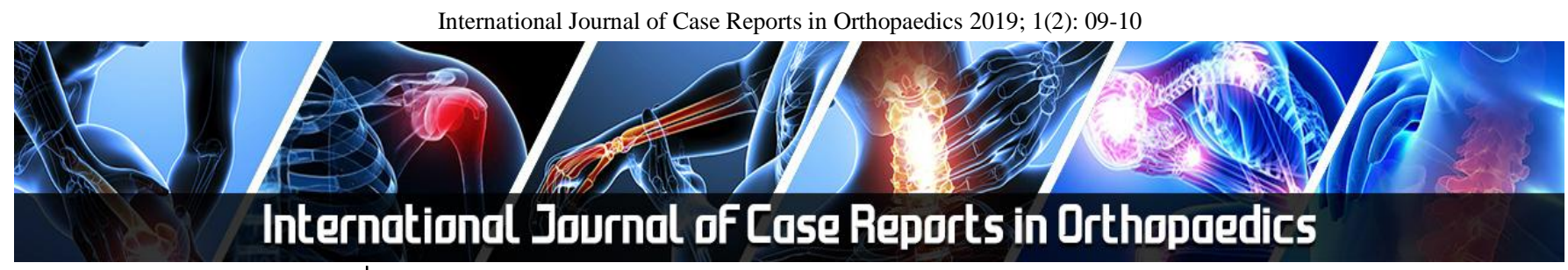

E-ISSN: 2707-8353 P-ISSN: 2707-8345 IJCRO 2019; 1(2): 09-10 Received: 15-05-2019 Accepted: 18-06-2019

Chanchal Rana

Department of Orthopaedics, National Institute of

Traumatology \& Orthopaedic Rehabilitation (NITOR), Dhaka, Bangladesh
Corresponding Author: Chanchal Rana

Department of Orthopaedics, National Institute of

Traumatology \& Orthopaedic Rehabilitation (NITOR), Dhaka, Bangladesh

\section{Frontal bone fracture- A case report in 28 years old male patient}

\section{Chanchal Rana}

DOI: https://doi.org/10.22271/27078345.2019.v1.i2a.10

Abstract

Frontal bone fractures offer significant challenges to surgeons and the treatment paradigm has been debated for many years. Acute concerns include protection of intracranial structures, identification of associated injuries and control of cerebrospinal fluid (CSF) leakage. We reported a case of frontal bone in 28 years old male patient managed with stainless steel wire.

Keywords: frontal bone fracture, cerebrospinal fluid, stainless steel

\section{Introduction}

Fractures involving the frontal region represent some of the least common injuries that affect the facial skeleton. The incidence is $5-15 \%$ of all facial fractures. However, because the frontal area makes up part of the brain case, the fatal potential of these injuries is much greater than with other facial fractures. A thorough neurologic examination is extremely important ${ }^{[1]}$.

Frontal bone fractures offer significant challenges to surgeons and the treatment paradigm has been debated for many years. Acute concerns include protection of intracranial structures, identification of associated injuries and control of cerebrospinal fluid (CSF) leakage. The aesthetic forehead contour is also an important consideration in repair. Past surgical modalities that removed the anterior bony frontal surface left life-long disfiguring defects and have been largely replaced by techniques that leave a smooth contour without visible scars. The frontal sinus is in close proximity to several intracranial structures. The posterior wall forms the anterior wall of the cranial vault and the floor of the frontal sinus contributes to the anterior superior roof of the orbit ${ }^{[2]}$.

Significant intracranial injury occurs more commonly with injury to the frontal sinus (12$17 \%$ of the time) than with injury to the mandible or mid face due to the proximity of the frontal sinus to the brain and the great forces required to cause a frontal sinus fracture ${ }^{[3]}$. Neurosurgical consultation should be obtained promptly if abnormal neurologic studies or brain CT changes are observed. Changes in mental status as well as nausea or vomiting are suggestive of intracranial injury. It is important to search for cerebrospinal fluid leakage from the nose or directly from lacerations and exposed bone which could signify dural displacement coupled with a posterior table fracture. A traumatic CSF fistula of the frontal sinus is a life threatening process deserving urgent attention ${ }^{[4]}$. We reported a case of frontal bone in 28 years old male patient managed with stainless steel wire.

\section{Case report}

A 28 years old male patient visited the department of Orthopedics with pain in frontal bone region since 5 days. History revealed that patient had fall 5 days back and got injury to frontal bone. There was severe pain, swelling in the same region. Patient medical history was non- contributory. Patient was subjected to CT scan which showed fractured line in frontal bone. The fracture found to be Undisplaced. The coronal incision was used to gain access to the fractured frontal bone. The incision was placed $5-7 \mathrm{~cm}$ behind the hairline and extended inferiorly to the level of the auricular helix. A wave like incision design was used as the scars would be less noticeable especially when the hair is wet. Fractured segments were repositioned after removing infected sinus lining and fixation was done and fixed in position with wires. Patient was recalled after 4 weeks. Post operative radiographs showed union without displacement of the segment. Prognosis of the treatment was good. 


\section{Discussion}

Fractures of the upper face and anterior skull base are a challenging neurosurgical, plastic, maxillofacial surgery problem. After clinical and radiographic evaluation of the fracture, prompt surgical intervention should be immediately instituted to excise any necrotic tissues inside or outside the cranial cavity, brain isolation by meticulous dural closure ablation of the frontal air sinuses and bony coverage of the region by either immediate or delayed frontal bone reconstruction ${ }^{[5]}$.

If frontal bone is comminuted, it is difficult to replace the small bony fragment by rigid bone plate fixation ${ }^{[6]}$. In such cases, it is prudent to leave the bony fragments where they are and camouflage the defect. Fractures of the frontal bone with associated involvement of the frontal sinuses (FSs) are relatively uncommon injuries in maxillofacial trauma, comprising just around $5 \%$ of all maxillofacial injuries. However, because of their location and close proximity to vital structures such as the orbital and intracranial contents, these injuries can have devastating sequelae if inadequately handled or improperly managed. Further, unaddressed frontal bone fractures with residual defects can leave a patient with disfiguring forehead deformities and prominent contour irregularities ${ }^{[7]}$. We reported a case of frontal bone in 28 years old male patient managed with stainless steel wire.

FS injuries involve fractures of the frontal bone with associated involvement of the FS to varying degrees. These injuries present quite a few challenges to the treating surgeon, and the ideal treatment paradigms have been debated over many years. Also of significance is the fact that as the FS is an air space containing microbial flora, that communicates with the nasal cavity, i.e., the exterior, hence FS fractures are open/compound and are prone to infections which can be potentially life-threatening ${ }^{[8]}$.

The peculiarity of frontal bone fractures is that a wrong choice or inadequate treatment could not only encompass functional or aesthetical problems but also more dangerous complications such as the risk of infections like meningitis, mucocele, encephalitis and cerebral abscess. Hence the necessity to recognize precociously and rightly the type of fracture and the intervening involvement of the adjacent structures in order to perform a proper surgical treatment according to the specific case, thus reducing the risk of infectious-related complications and either functional or aesthetical alterations at minimum ${ }^{[9]}$.

Jayaraj et al. ${ }^{[10]}$ suggested that effective and satisfactory results could be achieved in cases of significantly displaced inner and outer table fractures of the Frontal sinus by a more conservative protocol comprising of open reduction and internal fixation carried out via the existing scar of injury, without having to resort to the more radical intracranial approach and sinus cranialization. Nevertheless, presence of complicating factors such as cerebrospinal fluid rhinorrhea, evidence of meningitis or the development of encephalomeningocoeles necessitated the standard protocol of sinus exploration and its cranialization or obliteration.

\section{Conclusion}

Authors found that frontal bone fracture is common among road traffic accident. The use of stainless steel wire may be useful in undisplaced frontal bone fracture.

\section{References}

1. Salentijn EG, Peerdeman SM, Boffano $P$, van den Bergh B, Forouzanfar T. A ten-year analysis of the traumatic maxillofacial and brain injury patient in Amsterdam: Incidence and aetiology. J Craniomaxillofac Surg. 2014; 42:705-10.

2. Oannides $\mathrm{CH}$, Freihofer HP, Friens J. Fractures of the frontal sinus: a rationale of treatment. Br J Plastic Surg. 1993; 46:208-14.

3. Piotrowski WP. The primary treatment of frontobasal and midfacial fractures in patients with head injuries. $\mathbf{J}$ Oral Maxillofac Surg. 1992; 50:1264-8.

4. El Khatib K, Danino A, Malka G. The frontal sinus: A culprit or A victim? A review of 40 cases. J Craniomaxillofac Surg. 2004; 32(5):314-7.

5. Chen KT, Chen CT, Mardini S, Tsay PK, Chen YR. Frontal sinus fractures: A treatment algorithm and assessment of outcomes based on 78 clinical cases. Plast Recon Surg. 2006; 118:457-68.

6. Bell RB, Dierks EJ, Brar P, Potter JK, Potter BE. A protocol for the management of frontal sinus fractures emphasizing sinus preservation. J Oral Maxillofac Surg. 2007; 65(5):825-39.

7. Surya Rao RVM, Durairaj AR, Sundaramurthy N, Jesudasan JS. Frontal bone fractures - a report of three different methods of fixation: A case series. Int Surg J 2017; 4:3162-5.

8. Stanley RB, Jr Management of complications of frontal sinus and frontal bone fractures. Oper Tech Plastic Reconstr Surg. 1998; 5:296-301.

9. Chen TM, Wang HJ, Chen SL, Lin FH. Reconstruction of post-traumatic frontal-bone depression using hydroxyapatite cement. Ann Plast Surg. 2004; 52:3038.

10. Jeyaraj P. Frontal bone fractures and frontal sinus injuries: Treatment paradigms. Ann Maxillofac Surg 2019; 9:261-82. 